\title{
Improved Statistical TRE Model When Using a Reference Frame
}

\author{
Andrew D. Wiles and Terry M. Peters \\ Dept. of Medical Biophysics, The University of Western Ontario and \\ Imaging Research Laboratories, Robarts Research Institute, \\ London, Ontario, Canada
}

\begin{abstract}
Target registration error (TRE) refers to the uncertainty in localizing a point of interest after a point-based registration is performed. Common in medical image registration, the metric is typically represented as a root-mean-square statistic. In the late 1990s, a statistical model was developed based on the rigid body definition of the fiducial markers and the localization error associated in measuring the fiducials. The statistical model assumed that the fiducial localizer error was isotropic, but recently the model was reworked to handle anisotropic fiducial localizer error (FLE).

In image guided surgery, the statistical model is used to predict the surgical tool tip tracking accuracy associated with optical spatial measurement systems for which anisotropic FLE models are required. However, optical tracking systems often track the surgical tools relative to a patient based reference tool. Here the formulation for modeling the TRE of a surgical probe relative to a reference frame is developed mathematically and evaluated using a Monte Carlo simulation. The effectiveness of the statistical model is directly related to the FLE model, the fiducial marker design and the distance from centroid to target.
\end{abstract}

\section{Introduction}

The statistical model for the target registration error (TRE) associated with a point-based registration, assuming an isotropic and uncorrelated fiducial localizer error (FLE) model, was developed by Fitzpatrick et al. [1 in 1998. Based on the registration technique that solves the "orthogonal procrustes" problem using singular value decomposition, this model is a function of (i) the FLE (zero mean and standard deviation $\sigma$ on each cartesian axis), (ii) the fiducial marker rigid body definition $\left(\left\{\boldsymbol{x}_{i}\right\}\right.$ for $\left.i=1 \ldots N\right)$ and (iii) the target location $(\boldsymbol{r})$ in the rigid body space. The model was later extended to compute the independent variance components along the three orthogonal axes of the rigid body [2, and subsequently used to simulate the performance of optically tracked tools 3 .

Recently, we extended this formulation to accept a generalized FLE model 4 that was (i) isotropic or anisotropic and (ii) correlated or uncorrelated. The anisotropic FLE model is particularly important for optical tracking systems where the error is typically three times larger along one axis than the other two orthogonal axes. 
Registration algorithms exist that use the anisotropic FLE as a weighting factor in order to obtain a better estimate of the rigid transformation [5, 6]. Moreover, algorithms using the extended Kalman filter have been developed to predict the noise parameters associated with a point-based registration [7, 8. However, in both cases the algorithms are iterative in nature and may not be suitable when algorithm speed is critical and estimates of the TRE are required 1 . Therefore, a closed-form estimate of the TRE statistics associated with the closed-form point-based registration method is still relevant.

In [1] and 3], the FLE model was originally defined as a normally distributed random variable with zero mean and root-mean-square $(\mathrm{RMS})$ of $\mathrm{RMS}_{\mathrm{fle}}=3 \sigma^{2}$, or alternatively fle $\sim \mathcal{N}_{3}\left(\mathbf{0}, \sigma^{2} \boldsymbol{I}\right)$. In our improved model, the FLE was assumed to be a random variable with zero mean and specified covariance matrix, fle $\mathcal{N}_{3}(\mathbf{0}, \boldsymbol{\Sigma})$. Using tensor notation, the FLE is defined by the covariance $\sigma_{i j}$ in principal axes of the rigid body in a given orientation. The TRE mean goes to zero $\left(\mu_{\text {tre }}=0\right)$ while the covariance $\left(\Sigma_{\text {tre }}\right)$ and root-mean-square $\left(\mathrm{RMS}_{\text {tre }}\right)$ at $r$ are defined as

$$
\begin{gathered}
\left(\Sigma_{\text {tre }}(r)\right)_{i j} \approx \\
\epsilon^{2}\left(\frac{\sigma_{i j}}{N}+\sum_{k \neq i}^{K} \sum_{m \neq j}^{K} \frac{r_{k} r_{m}\left(\Lambda_{k k}^{2} \delta_{k m} \sigma_{i j}-\Lambda_{k k}^{2} \delta_{k j} \sigma_{i m}-\Lambda_{i i}^{2} \delta_{i m} \sigma_{k j}+\Lambda_{i i}^{2} \delta_{i j} \sigma_{k m}\right)}{\left(\Lambda_{k k}^{2}+\Lambda_{i i}^{2}\right)\left(\Lambda_{m m}^{2}+\Lambda_{j j}^{2}\right)}\right)
\end{gathered}
$$

$$
\begin{aligned}
\left\langle\left(\operatorname{RMS}_{\text {tre }}(r)\right)^{2}\right\rangle=\epsilon^{2}\left(\sum_{i=1}^{K} \frac{\sigma_{i i}}{N}\right. & +\sum_{i=1}^{K} \sum_{j \neq i}^{K} \frac{r_{j}^{2}\left(\Lambda_{j j}^{2} \sigma_{i i}+\Lambda_{i i}^{2} \sigma_{j j}\right)}{\left(\Lambda_{i i}^{2}+\Lambda_{j j}^{2}\right)^{2}} \\
& \left.+\sum_{i=1}^{K} \sum_{\substack { j \neq i \\
\begin{subarray}{c}{k \neq i \\
k \neq j{ j \neq i \\
\begin{subarray} { c } { k \neq i \\
k \neq j } }\end{subarray}}^{K} \frac{r_{j} r_{k} \Lambda_{i i}^{2} \sigma_{j k}}{\left(\Lambda_{i i}^{2}+\Lambda_{j j}^{2}\right)\left(\Lambda_{k k}^{2}+\Lambda_{i i}^{2}\right)}\right)
\end{aligned}
$$

where $\sigma_{i j}, \Sigma_{\text {tre }}$ are second order tensors in $\mathbb{R}^{3}$ and $\Lambda_{a a}$ are the $a$ th singular values of $X$ which consists of the $N$ demeaned fiducial marker positions in principal axes stacked in a $N \times K$ matrix $(K=3)$. $\epsilon$ is the smallness parameter used in perturbation theory and $\delta_{i j}$ is the Kronecker delta operator.

The new model formulation is limited to a single registration. In this paper, the model is extended to include the case where an optically tracked probe is measured relative to a reference tool. This was previously done by West and Maurer [3] for the TRE RMS statistic with the isotropic FLE model. Here, the TRE statistical model is extended to include the composition of transforms for the mean, covariance and RMS. Section 2 provides the mathematical derivation, while Section 3 provides details on the Monte Carlo simulation used to validate the model. The limitations of this model are discussed in Section 4 and Section 5 provides some concluding remarks.

\footnotetext{
${ }^{1}$ The algorithm speed criteria is highly dependent on the application.
} 


\section{Derivation of TRE by Composition of Transformations}

West and Maurer [3] showed that the TRE RMS for an optical probe measured relative to a reference tool is the sum in quadrature of the TRE RMS for the tool tip location in probe space and reference space. The mean, covariance and RMS are similarly derived here for for our generalized TRE model presented in [4].

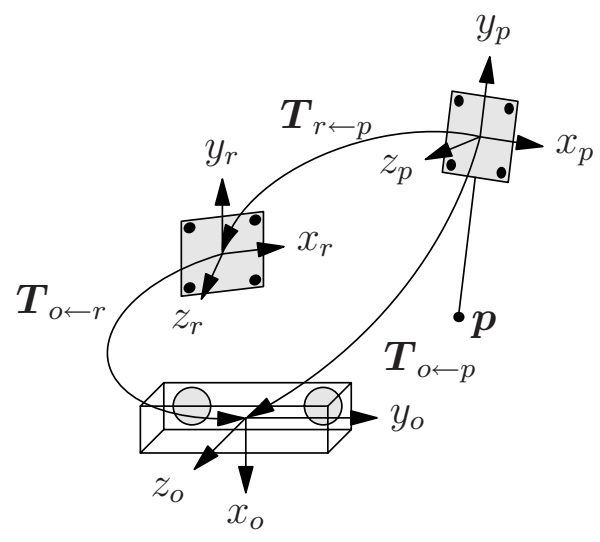

Fig. 1. Typical situation in image-guided surgery where probe $p$ is tracked by the optical tracking system $o$ relative to the reference tool $r$

Consider the case in Figure 1 where the tool tip of a probe is tracked by an optical tracking system relative to a reference tool. The tool tip is represented in probe, reference and optical tracker coordinate frames as $\boldsymbol{p}_{p}, \boldsymbol{p}_{r}$ and $\boldsymbol{p}_{o}$ respectively. In [4, the TRE is defined as the difference between the measured target location found via a point-based registration and the true (exact) target location. The definition is simplified by ignoring the gross transformations and only considering the erroneous portion of the transformation $\boldsymbol{T}_{e, p}$ (comprising rotation $\boldsymbol{R}_{e, p}$ and translation $\left.\boldsymbol{t}_{e, p}\right)$ in probe space2. The TRE at position $\boldsymbol{p}$ represented in probe space $p$ and associated with the registration of the fiducial markers in $p$ is given by

$$
\operatorname{tre}_{p}\left(\boldsymbol{p}_{p}\right)=\boldsymbol{p}_{p}-\boldsymbol{p}_{p}^{*}=\boldsymbol{T}_{e, p} \boldsymbol{p}_{p}^{*}-\boldsymbol{p}_{p}^{*}=\boldsymbol{R}_{e, p} \boldsymbol{p}_{p}^{*}+\boldsymbol{t}_{e, p}-\boldsymbol{p}_{p}^{*},
$$

where $\boldsymbol{p}_{p}^{*}$ and $\boldsymbol{p}_{p}$ are the exact and measured tool tip positions respectively.

From [3], it was shown the TRE for a combination of transforms is given by

$$
\begin{aligned}
\operatorname{tre}_{\mathrm{comb}}\left(\boldsymbol{p}_{r}\right) & =\boldsymbol{p}_{r}-\boldsymbol{p}_{r}^{*} \\
& =\boldsymbol{T}_{r \leftarrow o} \boldsymbol{p}_{o}-\boldsymbol{T}_{r \leftarrow o}^{*}\left(\boldsymbol{p}_{o}-\operatorname{tre}_{o \leftarrow p}\left(\boldsymbol{p}_{o}\right)\right) \\
& =\operatorname{tre}_{r}\left(\boldsymbol{p}_{r}\right)+\boldsymbol{R}_{r \leftarrow p}^{*} \operatorname{tre}_{p}\left(\boldsymbol{p}_{p}\right)
\end{aligned}
$$

\footnotetext{
${ }^{2}$ Probe space is assumed to be in principal axes.
} 
where equation (5) is the sum of reference TRE with the target at the "virtual tool tip" $\boldsymbol{p}_{r}$ and the probe TRE rotated into reference space. Both TRE values are computed in the principal axes and then transformed into the reference space. Since, the TRE represents a difference vector and not a spatial position, the transformation can be reduced to a rotation only.

In simulation, the exact transformations of the probe and reference tool are known, hence equation (5) can be used. However, if the formulation is used as a feedback mechanism during image-guided surgery then the exact transformations are no longer known but the TRE can be approximated using the measured transforms as shown in (6).

$$
\operatorname{tre}_{\mathrm{comb}}\left(\boldsymbol{p}_{r}\right) \approx \operatorname{tre}_{r}\left(\boldsymbol{p}_{r}\right)+\boldsymbol{R}_{r \leftarrow p} \operatorname{tre}_{p}\left(\boldsymbol{p}_{p}\right)
$$

\subsection{TRE Mean in a Reference Coordinate Frame}

The mean of the target registration error for a point measured relative to the reference is zero since the expectation of the target registration error vector is zero for each tool.

$$
\boldsymbol{\mu}_{\text {tre }, \text { comb }}\left(\boldsymbol{p}_{r}\right)=\left\langle\operatorname{tre}_{r}\left(\boldsymbol{p}_{r}\right)\right\rangle+\boldsymbol{R}_{r \leftarrow p}^{*}\left\langle\operatorname{tre}_{p}\left(\boldsymbol{p}_{p}\right)\right\rangle=\mathbf{0}
$$

\subsection{TRE Covariance in a Reference Coordinate Frame}

The covariance matrix is defined as:

$$
\boldsymbol{\Sigma}_{\text {tre }, \text { comb }}\left(\boldsymbol{p}_{r}\right)=\left\langle\left(\operatorname{tre}_{\mathrm{comb}}\left(\boldsymbol{p}_{r}\right)\right)\left(\operatorname{tre}_{\mathrm{comb}}\left(\boldsymbol{p}_{r}\right)\right)^{T}\right\rangle .
$$

Substitute (6) into (8) and expand to obtain

$$
\begin{aligned}
\boldsymbol{\Sigma}_{\text {tre }, \text { comb }}\left(\boldsymbol{p}_{r}\right)=\left\langle\left(\operatorname{tre}_{r}\left(\boldsymbol{p}_{r}\right)\right)\right. & \left.\left(\operatorname{tre}_{r}\left(\boldsymbol{p}_{r}\right)\right)^{T}\right\rangle-2\left\langle\left(\operatorname{tre}_{r}\left(\boldsymbol{p}_{r}\right)\right) \boldsymbol{R}_{r \leftarrow p}^{*} \operatorname{tre}_{p}\left(\boldsymbol{p}_{p}\right)^{T}\right\rangle \\
+ & \boldsymbol{R}_{r \leftarrow p}^{*}\left\langle\left(\operatorname{tre}_{p}\left(\boldsymbol{p}_{p}\right)\right)\left(\operatorname{tre}_{p}\left(\boldsymbol{p}_{p}\right)\right)^{T}\right\rangle \boldsymbol{R}_{r \leftarrow p}^{* T} .
\end{aligned}
$$

The expectations in the first and third terms reduce to the covariance matrix computed using equation $(11)$ for each respective rigid body registration with the target at $\boldsymbol{p}$. The expectation in the middle term goes to zero since the two TRE random variables are mutually independent resulting in a zero cross-covariance term.

$$
\boldsymbol{\Sigma}_{\text {tre }, \text { comb }}\left(\boldsymbol{p}_{r}\right)=\boldsymbol{\Sigma}_{\text {tre }, r}\left(\boldsymbol{p}_{r}\right)+\boldsymbol{R}_{r \leftarrow p} \boldsymbol{\Sigma}_{\text {tre }, p}\left(\boldsymbol{p}_{p}\right) \boldsymbol{R}_{r \leftarrow p}^{T}
$$

${ }^{3}$ Vector-matrix notation is used here for simplicity but the tensor notation used in

(1) is needed for implementation within any simulation code. 


\subsection{TRE RMS in a Reference Coordinate Frame}

The RMS for the combined TRE is

$$
\left(\operatorname{RMS}_{\text {tre }, \text { comb }}\left(\boldsymbol{p}_{r}\right)\right)^{2}=\left\langle\left(\operatorname{tre}_{\text {comb }}\left(\boldsymbol{p}_{r}\right)\right)^{T}\left(\operatorname{tre}_{\text {comb }}\left(\boldsymbol{p}_{r}\right)\right)\right\rangle .
$$

Equation (6) is substituted into (11) where we expand the expression, use the mutual independence of the two TRE vectors to eliminate the middle term and obtain a similar expression as in 3 .

$$
\left(\operatorname{RMS}_{\text {tre }, \text { comb }}\left(\boldsymbol{p}_{r}\right)\right)^{2}=\left(\operatorname{RMS}_{\text {tre }, r}\left(\boldsymbol{p}_{r}\right)\right)^{2}+\left(\operatorname{RMS}_{\text {tre }, p}\left(\boldsymbol{p}_{p}\right)\right)^{2}
$$

Note that the rotation matrix disappears since the RMS is rotationally invariant.

\section{Monte Carlo Simulations}

Monte Carlo simulations were performed to validate the TRE model using combination of transformations. The simulations use the probe and reference tool designs in West and Maurer 3 . The probe fiducial markers were placed in a rectangular pattern where the height $A$ and width $B$ were set to $71 \mathrm{~mm}$ and $54 \mathrm{~mm}$, respectively. The tool tip is $85 \mathrm{~mm}$ away from the marker centroid on an axis that passes through the centroid and is parallel to the height dimension. The reference tool is a square geometry with a side length $\ell$ set to $32 \mathrm{~mm}$. Eight different simulations were defined whereby the FLE Model and the distance between the reference and tool tip were varied as shown in Table 1 .

The isotropic and anisotropic FLE models were defined to have the same RMS statistic $\left(\mathrm{RMS}_{\mathrm{fle}}=1 / 3 \mathrm{~mm}\right)$ but the standard deviation along the $z$ axis was three times larger than the $x$ and $y$ axes in the anisotropic FLE model. The tool tip position was fixed at a specified location in the volume and the probe orientation was randomly selected under the restriction that the orientation was relevant in terms of an optical tracking situation. Similarly, the reference tool position and orientation were randomly selected so that the reference tool was at a distance $d$ from the tool tip. After analyzing the results from the initial eight test cases, another four were simulated with the size of the reference tool increased from $32 \mathrm{~mm}$ to $64 \mathrm{~mm}$.

In each case, $M=1000$ random tool orientations were tested. For each random tool orientation, $N=100,000$ sample measurements were simulated to obtain the TRE statistics. The predicted statistics were computed for each of the $M$ random tool orientations using equations (7), (10) and (12) respectively.

Two forms of analysis were performed on the resulting data. First, hypothesis tests were used to determine whether the predicted mean and covariance matched the simulated statistics $(\alpha=0.05)$. The hypothesis tests were performed using the likelihood ratio function and the Wishart distribution (see [9,4). Then the percent differences 4 between the predicted and simulated RMS results were examined. The summary of results are presented in Table 1.

\footnotetext{
$4 \%$ diff $=100\left(\mathrm{RMS}_{\text {theory }}-\mathrm{RMS}_{\text {sim }}\right) / \mathrm{RMS}_{\text {sim }}$
} 
Table 1. Monte Carlo simulation results for 12 cases where the FLE model, reference body size and the distance to the rigid body are varied. The hypothesis tests use the likelihood ratio and Wishart distribution [9]. Null Hypothesis for Test \#1 is $H_{0}$ : $\boldsymbol{\Sigma}_{\text {sim }}=\boldsymbol{\Sigma}_{\text {tre }}$ and Test $\# 2$ is $H_{0}: \boldsymbol{\mu}=\mathbf{0}, \boldsymbol{\Sigma}_{\text {sim }}=\boldsymbol{\Sigma}_{\text {tre }}$.

\begin{tabular}{|c|c|c|c|c|c|c|}
\hline \multirow[t]{2}{*}{$\overline{\overline{\text { Case }}}$} & \multirow[t]{2}{*}{$\begin{array}{c}\text { FLE } \\
\text { Model } \\
\end{array}$} & \multirow{2}{*}{$\begin{array}{c}\text { Ref. } \\
\text { Size } \\
\ell\end{array}$} & \multirow{2}{*}{$\begin{array}{c}\begin{array}{c}\text { Working } \\
\text { Distance }\end{array} \\
d\end{array}$} & \multicolumn{2}{|c|}{\begin{tabular}{|c|} 
Hypothesis Test \\
Percent Accepted
\end{tabular}} & $\begin{array}{l}\text { RMS Percent Diff. } \\
\text { Summary Statistics }\end{array}$ \\
\hline & & & & $\# 1$ & $\# 2$ & $\begin{array}{llll}\text { Mean } & \text { S.D. } & \text { Min } & \text { Max }\end{array}$ \\
\hline 1 & Isotropic & $32 \mathrm{~mm}$ & $100 \mathrm{~mm}$ & $94.8 \%$ & $98.2 \%$ & $-0.01 \% 0.15 \%-0.47 \% \quad 0.46 \%$ \\
\hline 2 & Isotropic & $32 \mathrm{~mm}$ & $200 \mathrm{~mm}$ & $94.6 \%$ & $86.0 \%$ & $0.00 \% \quad 0.17 \%-0.52 \% \quad 0.60 \%$ \\
\hline 3 & Isotropic & $32 \mathrm{~mm}$ & $300 \mathrm{~mm}$ & $89.0 \%$ & $39.2 \%$ & $0.00 \% \quad 0.17 \%-0.52 \% \quad 0.56 \%$ \\
\hline 4 & Isotropic & $32 \mathrm{~mm}$ & $400 \mathrm{~mm}$ & $79.2 \%$ & $4.20 \%$ & $0.00 \% \quad 0.16 \%-0.50 \% \quad 0.54 \%$ \\
\hline 5 & Anisotropic & $32 \mathrm{~mm}$ & $100 \mathrm{~mm}$ & $95.3 \%$ & $99.7 \%$ & $0.00 \% \quad 0.19 \%-0.52 \% \quad 0.62 \%$ \\
\hline 6 & Anisotropic & $32 \mathrm{~mm}$ & $200 \mathrm{~mm}$ & $71.4 \%$ & $25.2 \%$ & $-0.01 \% 0.20 \%-0.56 \% 0.67 \%$ \\
\hline 7 & Anis & $32 \mathrm{~mm}$ & $300 \mathrm{~mm}$ & $37.4 \%$ & $3.8 \%$ & $0.00 \% \quad 0.19 \%-0.82 \% \quad 0.83 \%$ \\
\hline 8 & Anisotropic & $32 \mathrm{~mm}$ & $400 \mathrm{~mm}$ & $16.0 \%$ & $0.0 \%$ & $0.01 \% \quad 0.20 \%-0.75 \% \quad 0.61 \%$ \\
\hline 9 & Anisotropic & $64 \mathrm{~mm}$ & $100 \mathrm{~mm}$ & $94.4 \%$ & $98.3 \%$ & $0.00 \% 0.19 \%-0.61 \% 0.64 \%$ \\
\hline 10 & Anis & $64 \mathrm{~mm}$ & $200 \mathrm{~mm}$ & $95.9 \%$ & $98.9 \%$ & $0.00 \% 0.19 \%-0.71 \% 0.65 \%$ \\
\hline 11 & Anisotropic & $64 \mathrm{~mm}$ & $300 \mathrm{~mm}$ & $93.8 \%$ & $91.4 \%$ & $0.01 \% \quad 0.19 \%-0.61 \% 0.62 \%$ \\
\hline 12 & Anisotropic & $64 \mathrm{~mm}$ & $400 \mathrm{~mm}$ & $93.5 \%$ & $80.7 \%$ & $0.00 \% \quad 0.20 \%-0.76 \% \quad 0.67 \%$ \\
\hline
\end{tabular}

\section{Discussion}

The results show two key findings. First, the acceptance of the hypothesis tests are significantly reduced as the distance $d$ between the tool tip and the reference tool is increased. The hypothesis testing was expanded to include similar tests for the probe and reference tools separately. It was found that the probe maintained a high rate of acceptance of the null hypothesis (typically larger than 90\%), but the reference tool exhibited a lower acceptance of the null hypothesis as the distance to the target increased. The predicted RMS percent differences agreed very well with the simulated results, but the percent differences may be misleading because the RMS results at $d=400 \mathrm{~mm}$ (e.g., Case 8 RMS is $2.5 \mathrm{~mm}$ to $4.4 \mathrm{~mm}$ ) will be significantly larger than the results at $100 \mathrm{~mm}$ (e.g., Case 5 RMS is $0.7 \mathrm{~mm}$ to $1.2 \mathrm{~mm}$ ). Hence a $1 \%$ difference in Case 8 is larger than a similar percentage in Case 5. Therefore, although the hypothesis tests and RMS look very different they may be exhibiting similar trends with varying degrees of sensitivity.

The second key finding was that if the rigid body size for the reference tool, i.e., $\ell$ increased to $64 \mathrm{~mm}$, the hypothesis tests accepted the null hypothesis at rates greater than $80 \%$ for all distances. By increasing the rigid body size, the moment of inertia of the rigid body is strengthened such that the rotational error is decreased given the same FLE model.

For both findings, it is suspected that the relationship between the target distance, rigid body size and the null hypothesis acceptance rate may be due to the rotational error in the registration. Recall from [1, 4] that a power series expansion was used to derive the TRE statistical model: $\boldsymbol{R}=\boldsymbol{I}+\epsilon \boldsymbol{R}^{(1)}+$ 
$O\left(\epsilon^{2}\right) \cdot \boldsymbol{R}^{(1)}$ is the erroneous portion of the transformation for the TRE, which is equivalent to $\boldsymbol{R}_{e, p}$ from equation (3). If we examine equations (11) and (2), it is clear that the TRE is normally distributed and formed by rotating, scaling and summing the contributions of the normally distributed FLE statistics. But if the error from (3) is separated into rotational and translational components, then we see that the portion of the error distribution attributed to the rotational error will lie on the surface of a sphere with a radius $\rho$ equal to the distance from the target to the marker centroid. This distribution, suspected to be approximated by the von Mises-Fisher (spherical) distribution [10, is not normal. But if the rotational error is small then the von Mises distribution is approximated by a 2D projected normal distribution. The TRE becomes a 3D error distribution when the translational error component is taken into account. Consider a case restricted to 2D cartesian space, if the span (or 95\% confidence interval) of the rotational error can be described by the angle $\theta$ that subtends from the mean to the error extents, then the path where the measured target may lie is related to the arc length $s=2 \rho \theta$. However, the span for the projected normal distribution is a chord for the arc $s$ given by $w=2 \rho \sin \theta$. (This can be extended to 3D cartesian space by using a surface area subtended by a solid angle.) The ratio of the two spans becomes $b=\sin \theta / \theta$ which suggests that the rotational error given by the TRE model is underestimated by the factor $b$. Since $\theta$ is typically small, the underestimation is negligible for small $\rho$. For example, if $\theta=2^{\circ}$ then $b=0.9998$ and the difference between $s$ and $w$ at $\rho=100 \mathrm{~mm}$ is approximately $0.0014 \mathrm{~mm}$. As $\rho$ increases, the underestimation becomes significant in terms of the Wishart based hypothesis tests but when used practically the difference between the simulated and predicted $95 \%$ confidence regions are not very different. In the future, an expression that defines the point at which the model significantly underestimates the true distribution would be useful.

In image registration applications, this is not a significant issue as the target is usually close to the centroid since it is typically contained within the convex hull formed by the fiducial markers. However, in image-guided surgery applications, such as those that track C-arms and fluoroscopes, it is common to track rigid bodies with targets that exist outside of the fiducial marker geometry at large distances such as $400 \mathrm{~mm}$ and greater. Therefore, it is possible that when maintaining the same FLE and rigid body geometry while increasing the target distance, as done in this paper, the TRE model will underestimate the actual TRE error. However, as shown in the RMS percent differences, and by the example given, this underestimation is very small and hence the model is a very good approximation.

\section{Conclusions}

Overall, the proposed model performs well. However, this is only true for situations when the reference tool has an appropriate combination of rotational error and closeness to the target since the TRE model underestimates the TRE span as a function of target distance. This issue is not as significant in image registration 
applications where the target is often contained within the convex hull formed by the fiducial markers, but becomes significant when this formulation is used in the simulation of optically tracked tools for image-guided surgery.

\section{Acknowledgements}

This work was supported by CIHR, NSERC, ORDCF, CFI, UWO and NDI.

\section{References}

1. Fitzpatrick, J.M., West, J.B., Maurer Jr., C.R.: Predicting error in rigid-body point-based registration. IEEE Trans. Med. Imag. 17(5), 694-702 (1998)

2. Fitzpatrick, J.M., West, J.B.: The distribution of target registration error in rigidbody point-based registration. IEEE Trans. Med. Imag. 20(9), 917-927 (2001)

3. West, J.B., Maurer Jr., C.R.: Designing optically tracked instruments for imageguided surgery. IEEE Trans. Med. Imag. 23(5), 533-545 (2004)

4. Wiles, A.D., Likholyot, A., Frantz, D.D., Peters, T.M.: Derivation of TRE using anisotropic FLE. Technical Report NDI-TR-0421, Northern Digital, Inc. Waterloo, Ontario, Canada, Contact: support@ndigital.com (2007)

5. Chu, M.T., Trendafilov, N.T.: On a differential equation approach to the weighted orthogonal Procrustes problem. Statistics and Computing 8, 125-133 (1998)

6. Batchelor, P.G., Fitzpatrick, J.M.: A study of the anisotropically weighted Procrustes problem. In: Proceedings of IEEE Workshop on Mathematical Methods in Biomedical Image Analysis, Hilton Head, SC, USA, June 2000, pp. 212-218. IEEE Computer Society Press, Los Alamitos (2000)

7. Pennec, X., Thirion, J.-P.: A framework for uncertainty and validation of 3-d registration methods based on points and frames. International Journal of Computer Vision 25(3), 203-229 (1997)

8. Nicolau, S., Pennec, X., Soler, L., Ayache, N.: An accuracy certified augmented reality system for therapy guidance. In: Pajdla, T., Matas, J(G.) (eds.) ECCV 2004. LNCS, vol. 3023, pp. 79-91. Springer, Heidelberg (2004)

9. Anderson, T.W.: An Introduction to Multivariate Statistical Analysis. John Wiley \& Sons, Toronto, Ontario (1984)

10. Mardia, K.V., Jupp, P.E.: Directional Statistics. John Wiley \& Sons, Toronto (2000) 